\title{
A SIMPLIFIED TREATMENT OF THE REGULAR SINGULAR POINT*
}

BY

\section{GEORGE D. BIRKHOFF}

The usual treatment of regular singular points involves the substitution of an infinite series in the differential equation; the series thus formally determined is then proved to be convergent. It is possible to replace this part of the work by a simpler and more direct argument, and this is given below in IV, along with an outline of the entire discussion. $\dagger$ The idea employed is quite similar to that used by Liapounoff in another connection (see PiCARD, Traité d'Analyse, vol. 3, pp. 362-363). The analysis is made in full only for a single equation of the second order, and afterward the generalization is indicated.

I. We will assume that it has been proved, say by the method of successive approximations, that the general solution of

$$
y^{\prime \prime}+p(x) y^{\prime}+q(x) y=0
$$

at a point of the $x$-plane where $p(x)$ and $q(x)$ are single-valued and analytic, has the form

$$
y=c_{1} y_{1}+c_{2} y_{2} \text {, }
$$

in which $y_{1}$ and $y_{2}$ are linearly independent and analytic at the point.

II. Now let $x=a$ be an isolated singular point of one or both of the singlevalued analytic functions $p(x)$ and $q(x)$, so that by definition $x=a$ is a singular point of the differential equation. Consider the solutions $y$ of (1) in a certain vicinity of $x=a$ which is taken so small as to contain no further singular points; these solutions are analytic except perhaps at $x=a$, by $\mathrm{I}$. If we make a positive circuit of $x=a$ the solutions will undergo a linear substitution, which we will write in the form

$$
\begin{aligned}
& \bar{y}_{1}=c_{1} y_{1}+c_{2} y_{2}, \\
& \bar{y}_{2}=d_{1} y_{1}+d_{2} y_{2} .
\end{aligned}
$$

There will exist multiplicative solutions, $\bar{y}=\rho y$, for values of $\rho$ such that

$$
\left|\begin{array}{cc}
c_{1}-\rho & d_{1} \\
c_{2} & d_{2}-\rho
\end{array}\right|=0 .
$$

* Presented to the Society, February 26, 1910.

† SCHLESINGER has avoided this substitution (Journal für die reine und angevandte Mathematik, vol. 132 (1907), pp. 247-254) but his method is not nearly so simple as the one here presented. 
Thus we are led to the conclusion that there are two linearly independent solutions of the form

$$
\begin{array}{ll}
y_{1}=(x-a)^{\kappa_{1}} f_{1}(x), & \kappa_{1}=\frac{\log \rho_{1}}{2 i \pi}, \\
y_{2}=(x-a)^{\kappa_{2}} f_{2}(x), & \kappa_{2}=\frac{\log \rho_{2}}{2 i \pi} ;
\end{array}
$$

or in a degenerate case, $\rho_{1}=\rho_{2}$, of the form

$$
y_{1}=(x-a)^{\kappa_{1}} f_{1}(x), \quad y_{2}=y_{1} \log (x-a)+(x-a)^{\kappa_{1}} f_{2}(x) ;
$$

here $f_{1}$ and $f_{2}$ are single-valued and analytic in the neighborhood of $x=a$, and can therefore be expanded in Laurent series at this point.

III. Regular solutions are now defined as solutions of the form (2), (3) in which $f_{1}, f_{2}$ are either analytic or have a pole at $x=a$. A necessary condition that the solutions of (1) be regular at $x=a$ is found at once to be that $p(x)$ and $q(x)$ cannot become infinite to higher than the first and second orders respectively at $x=a$, by writing (1) in the form

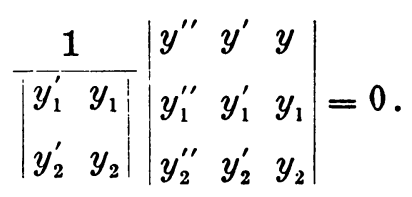

If the coefficients $p(x)$ and $q(x)$ have this character at $x=a$, that point is called a regular singular point of (1).

IV. Conversely, at a regular singular point the solutions must be regular. For write

$$
z=(x-a) y^{\prime} .
$$

The differential equation (1) has as its equivalent the equations

$$
\begin{aligned}
& y^{\prime}=\frac{z}{x-a}, \\
& \begin{aligned}
z^{\prime}=(x-a) y^{\prime \prime}+y^{\prime}=-(x-a)\left[p(x) y^{\prime}+q(x) y\right]+y^{\prime} \\
\quad=-(x-a) q(x) y+\left(\frac{1}{x-a}-p(x)\right) z .
\end{aligned}
\end{aligned}
$$

Consider now thereal positive function

$$
U=\left|y^{2}\right|+\left|z^{2}\right|
$$

for values of $x$ on any ray emanating from $x=a$, and denote the distance $|x-a|$ by $r$. Since, if $y=\alpha+i \beta$, one has $\left|y^{2}\right|=\alpha^{2}+\beta^{2}$, it is apparent that both terms of $U$, and therefore $U$ also, are continuous functions of $r$ with continuous $r$-derivatives. Moreover the inequality 


$$
\left|\frac{\partial U}{\partial r}\right| \leqq\left|2 y y^{\prime}\right|+\left|2 z z^{\prime}\right|
$$

holds, inasmuch as, for any function $w$ which is analytic, one has

$$
\begin{gathered}
w=R e^{\Theta i}, \quad R=|w|, \\
\frac{\partial w}{\partial r}=e^{\Theta i}\left[\frac{\partial R}{\partial r}+i R \frac{\partial \Theta}{\partial r}\right], \quad w^{\prime}=\frac{d w}{d x}=\frac{1}{e^{\theta i}} \frac{\partial w}{\partial r},
\end{gathered}
$$

and hence

$$
\left|\frac{\partial|w|}{\partial r}\right| \leqq\left|\frac{\partial w}{\partial r}\right|=\left|w^{\prime}\right|
$$

But in the two equations (4) the coefficients of $y$ and $z$ have poles of at most the first order at $x=a$, so that clearly one has

$$
\left|y^{\prime}\right|,\left|z^{\prime}\right| \leqq \frac{M}{r}\{|y|+|z|\},
$$

where $M$ is independent of the particular ray under consideration. Accordingly there is obtained

This may be written

$$
\left|\frac{\partial U}{\partial r}\right| \leqq \frac{2 M}{r}\{|y|+|z|\}^{2} \leqq \frac{4 M U}{r}
$$

$$
-\frac{4 M}{r} \leqq \frac{\partial \log U}{\partial r} \leqq \frac{4 M}{r}
$$

Integrating between $r$ and $r_{0}\left(r \leqq r_{0}\right)$ one obtains

$$
-4 M \log \frac{r_{0}}{r} \leqq \log \frac{U_{0}}{U} \leqq 4 M \log \frac{r_{0}}{r}
$$

so that

$$
\left(\frac{r_{0}}{r}\right)^{-4 M} \leqq \frac{U_{0}}{U} \leqq\left(\frac{r_{0}}{r}\right)^{4 M}
$$

This gives the inequality

$$
U \leqq U_{0}\left(\frac{r}{r_{0}}\right)^{-4 M}
$$

Now $U_{0}$ is the value of $U$ at a distance $r_{0}$ from $x=a$. Let $G$ denote the upper limit of such values for $0 \leqq \arg (x-a) \leq 2 \pi$. Then we have

$$
U \leqq G\left(\frac{r}{r_{0}}\right)^{-4 . M}, \quad[0 \leqq \arg (x-a)<2 \pi] .
$$

By definition of $U$ it is clear then that

$$
|y| \leqq v \bar{G}\left(\begin{array}{c}
r \\
r_{0}
\end{array}\right)^{-2 M} \quad[0 \leqq \arg (x-a)<2 \pi],
$$


i. e., the branch of $y$ corresponding to the condition $0 \leqq \arg (x-a)<2 \pi$ becomes infinite only to a finite order at $x=a$.

From equations (2) and (3) it is now concluded that the single-valued functions $f_{1}$ and $f_{2}$, analytic except perhaps at $x=a$, have also this property. Hence, a positive integer $m$ can be chosen so large that $(x-a)^{m} f_{1}$ and $(x-a)^{m} f_{2}$ are finite in the neighborhood of $x=a$. By Riemann's theorem these functions are analytic at $x=a$. Therefore $f_{1}$ and $f_{2}$ are analytic or have poles at $x=a$, which we desired to prove.

V. The formal determination of the series (2) and (3) still remains to be made, but for many purposes this expansion is not necessary. The usual discussion shows that there exist essentially two and only two expansions (2) or (3) which formally satisfy (1), and these must, of course, coincide with the desired series.

Part IV of the above treatment (as well as the remainder) admits of extension to a single equation of the $n$th order in $y_{1}$, or $n$ equations of the first order in $y_{1}, y_{2}, \cdots, y_{n}$; in fact the first case is always reduced to the second by a substitution

$$
y_{2}=(x-a) y_{1}^{\prime}, \quad y_{3}=(x-a)^{2} y_{1}^{\prime \prime}, \cdots .
$$

If we write the linear system as follows:

$$
\frac{d y_{i}}{d x}=\sum_{j=1}^{n} a_{i j}(x) y_{j} \quad(i=1,2, \cdots, n),
$$

the coefficients $a_{i j}(x)$ have then at most poles of the first order at $x=a$ if $x=a$ is a regular singular point. Hence one finds in a certain vicinity of $x=a$

$$
\left|a_{i j}(x)\right| \leqq \frac{M}{r} .
$$

If one considers the positive real function

one finds

$$
U=\sum_{i=1}^{n}\left|y_{i}^{2}\right|
$$

$$
\begin{aligned}
\left|\frac{\partial U}{\partial r}\right| & \leqq \sum_{i=1}^{n}\left|2 y_{i} y_{i}^{\prime}\right| \\
& \leqq \frac{2 M}{r}\left(\sum_{i=1}^{n}\left|y_{i}\right|\right)^{2} . \\
& \leqq \frac{2 n M}{r} U .
\end{aligned}
$$

The remainder of the argument is now made as before.

Princeton University. 